\title{
Direct Imaging of Radionuclide-Produced Electrons and Positrons with an Ultrathin Phosphor
}

\author{
Liying Chen ${ }^{1}$, Lisa S. Gobar ${ }^{1}$, Negar G. Knowles ${ }^{1}$, Zhonglin Liu ${ }^{1}$, Arthur F. Gmitro ${ }^{1,2}$, and Harrison H. Barrett ${ }^{1-3}$ \\ ${ }^{I}$ Department of Radiology, University of Arizona, Tucson, Arizona; ${ }^{2}$ College of Optical Sciences, University of Arizona, Tucson, \\ Arizona; and ${ }^{3}$ Applied Mathematics Program, University of Arizona, Tucson, Arizona
}

\begin{abstract}
Current electron detectors are either unable to image in vivo or lack sufficient spatial resolution because of electron scattering in thick detector materials. This study was aimed at developing a sensitive high-resolution system capable of detecting electronemitting isotopes in vivo. Methods: The system uses a lenscoupled charge-coupled-device camera to capture the scintillation light excited by an electron-emitting object near an ultrathin phosphor. The spatial resolution and sensitivity of the system were measured with a $3.7-\mathrm{kBq}{ }^{90} \mathrm{Y} / 90 \mathrm{Sr} \beta$-source and a $70-\mu \mathrm{m}$

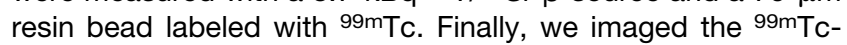
pertechnetate concentration in the mandibular gland of a mouse in vivo. Results: Useful images were obtained with only a few hundred emitted $\beta$ particles from the ${ }^{90} \mathrm{Y} /{ }^{90} \mathrm{Sr}$ source or conversion electrons from the ${ }^{99 m}$ Tc bead source. The in vivo image showed a clear profile of the mandibular gland and many fine details with exposures of as low as $30 \mathrm{~s}$. All measurements were consistent with a spatial resolution of about $50 \mu \mathrm{m}$, corresponding to 2.5 detector pixels with the current camera. Conclusion: Our new electron-imaging system can image electron-emitting isotope distributions at high resolution and sensitivity. The system is useful for in vivo imaging of small animals and small, exposed regions on humans. The ability to image $\beta$ particles, positrons, and conversion electrons makes the system applicable to most isotopes.
\end{abstract}

Key Words: electron imaging; CCD; phosphor; in vivo imaging; positron

J Nucl Med 2008; 49:1141-1145

DOI: 10.2967/jnumed.107.040568

\section{D}

irect imaging of electron emissions from radionuclides is valuable in biodistribution and microdosimetry studies, but most current electron detectors are unable to image in vivo because of either the physical configuration or insufficient sensitivity. Others have either compromised spatial resolution or limited sensitivity due to their design tradeoffs; thin detector materials lack sensitivity, and thick materials cause sufficient scatter of the electrons to impair resolution.

Received Aug. 1, 2007; revision accepted Mar. 19, 2008.

For correspondence or reprints contact: Liying Chen, Department of Radiology, University of Arizona, P.O. Box 245067, Tucson, AZ 85724.

E-mail: lichen@email.arizona.edu

COPYRIGHT @ 2008 by the Society of Nuclear Medicine, Inc.
Film-based autoradiography is capable of micrometerscale resolution because of the thin emulsions used, but film is limited by its narrow linear response range and relatively low sensitivity, requiring long exposure times. Digital autoradiography systems improve linearity and sensitivity over film with a variety of thicker detector materials.

Arrays of gas detectors with dedicated readout electronics can provide spatial information (1-4). One of the better systems, the Beta Imager 2000 (LabLogic Systems Limited), has been reported to achieve 100- to $300-\mu \mathrm{m}$ resolution.

Double-sided silicon detectors with orthogonal readout electrodes can achieve $115 \mu \mathrm{m}$ in full width at half maximum (FWHM) (5). Other solid-state detectors used for this application include charge-coupled devices (CCD), complementary metal-oxide semiconductor arrays, and hybrid detector technologies (6-8). Their limited sensitivity usually requires tens of hours per exposure.

Scintillators and phosphors can be used in combination with digital light sensors such as CCD and complementary metal-oxide semiconductor cameras. One approach to achieve sufficient sensitivity is to use thick scintillators to improve light production (9-11), and another is to amplify the excited light signals, such as by multiple microchannel plates (12-14). Photostimulable phosphor plates can store the deposited energy of electrons, and an image is later read out digitally by laser scanning. Intraoperative $\beta$-imaging probes have recently been developed using scintillators; Hoffman et al. described one based on a microcolumnar CsI(Tl) scintillator and a fiberoptic bundle (15).

The system described in the present paper uses a lens to couple the light produced by an ultrathin phosphor directly to a CCD. Though almost all of the electrons pass through the phosphor, each deposits some small portion of its kinetic energy there and produces light. With modern CCDs having large sensors, low noise, and high quantum efficiency, and with lenses of high effective numeric aperture, this tiny amount of light is sufficient for $\beta$ imaging with excellent sensitivity and resolution. Many $\beta$ detectors described in the literature $(4,8,16,17)$ are based on the assumption that it is necessary to absorb most or all of the electron energy in the detector. This leads to detector thicknesses and pixel sizes on the order of $1 \mathrm{~mm}$. By 
contrast, the approach described here uses a much thinner detector and consequently gets far better spatial resolution in situations where the detector can be placed in contact with the object. Little or no loss in counting efficiency results from the thin detector if the optical system is sufficiently sensitive.

\section{MATERIALS AND METHODS}

\section{System Configuration}

The CCD (VersArray 1300B; Roper Scientific) in our electronimaging system has $1,300 \times 1,340$ pixels, each $20 \mu \mathrm{m} \times 20 \mu \mathrm{m}$. The detecting area of the CCD chip is thus about $25 \mathrm{~mm} \times 25 \mathrm{~mm}$. The CCD is cooled to $-100^{\circ} \mathrm{C}$, at which temperature its dark current is essentially negligible (4 electrons/h) (18). Its readout noise level is also reduced to 4 electrons per pixel at the slower readout speed of $50 \mathrm{kHz}(18)$.

To achieve the necessary optical coupling efficiency, we use 2 F/1.2 camera lenses of 50-mm focal length in a telecentric configuration shown in Figure 1. The first lens collimates the light from a point source, and the second refocuses it to the detector. The resulting magnification is unity, and the light-collecting cone is as large as it can be with these lenses; if a single F/1.2 lens were used at unit magnification, the effective F-number would be 2.4, and the coupling efficiency would be reduced by a factor of 4 .

The ultrathin phosphor consists of a monolayer of 3- $\mu \mathrm{m} \mathrm{P} 47$ phosphor powder coated on a 3- $\mu$ m-thick clear Mylar foil (Applied Scintillation Technologies). The phosphor is always placed near the radioactive object being imaged, usually in contact.

The entire system is enclosed in a light-tight box. A white-light source can be coupled into the box from outside whenever necessary. When the white-light source is turned off, the ambient background in the box is negligible (18).

\section{System Performance}

We first imaged a $3.7-\mathrm{kBq}{ }^{90} \mathrm{Y} /{ }^{90} \mathrm{Sr}$ standard $\beta$ source (Isotope Products Lab) produced by evaporating a radioactive salt solution

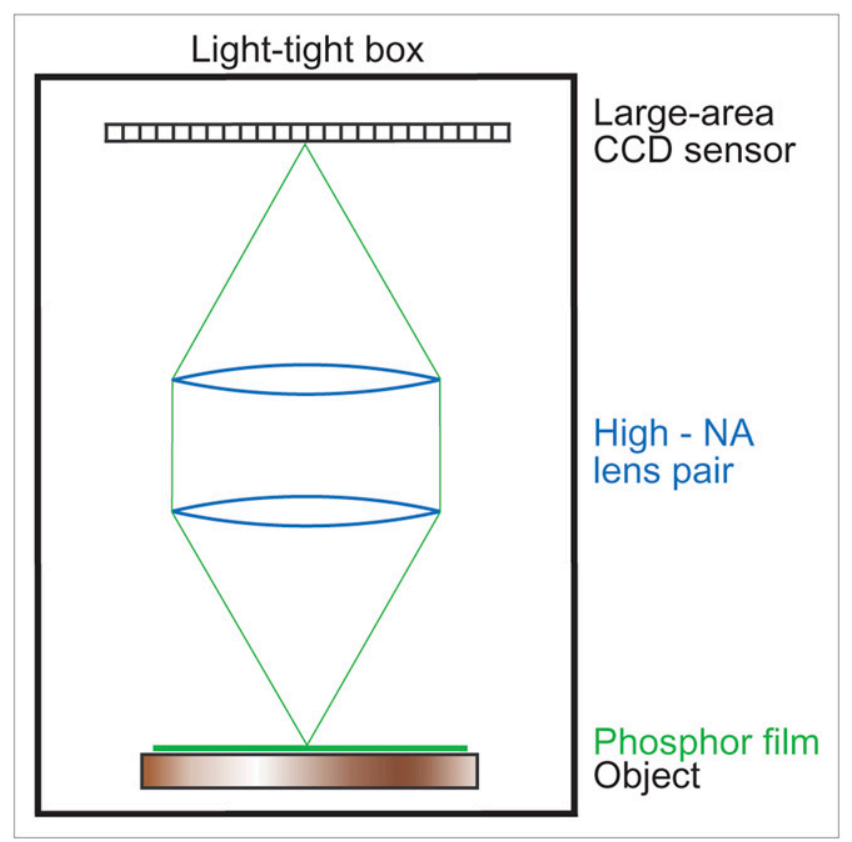

FIGURE 1. Schematic layout of our electron-imaging system. on a 3-mm-diameter area of a 23.8-mm-diameter 6.4- $\mu$ m-thick Mylar foil. A second identical Mylar foil was used as a cover slip, and the 2 were clamped together by a removable aluminum ring holder seen in Figure 2A. In our experiments, the ultrathin phosphor was placed directly on the surface of the cover slip.

We then imaged a 70- $\mu \mathrm{m}$-diameter anion-exchange resin bead (BioRad), with $814 \mathrm{~Bq}$ of ${ }^{99 \mathrm{~m}} \mathrm{Tc}$ attached to it. A micrograph of the dried bead under a regular microscope is shown in Figure 3A. During imaging, the bead was placed on a glass slide, covered with a phosphor.

Finally, we imaged a very narrow tungsten slit using the same optical imaging components in the electron-imaging system. The nominal width of the slit is $10 \mu \mathrm{m}$. A white-light source illuminated the slit from the bottom. During imaging, there was no phosphor on the slit top, and no radioactive source was used.

\section{In Vivo Imaging}

In this experiment, we imaged in vivo an anesthetized normal mouse $30 \mathrm{~min}$ after intravenous administration of $74 \mathrm{MBq}$ of ${ }^{99 \mathrm{~m} T c-p e r t e c h n e t a t e ~(w h i c h ~ a c c u m u l a t e s ~ i n ~ g l a n d u l a r ~ t i s s u e s) ~}$ in $0.2 \mathrm{ml}$ of solution. Before imaging, the overlying skin on the mouse chest was retracted to expose its mandibular glands. During imaging, we placed an ultrathin phosphor and then a glass slide on the surface of the glands. Pressure was applied to flatten the surface. The animal's body temperature was regulated by a heating pad. Exposure times ranged from 0.5 to $5 \mathrm{~min}$.

\section{RESULTS}

\section{Resolution}

Figures $2 \mathrm{~B}$ and $2 \mathrm{C}$ show the electron images of the ${ }^{90} \mathrm{Y} /{ }^{90} \mathrm{Sr}$ source. Although the source was manufactured to be a point source, imaging by our high-resolution system reveals an annular distribution of the radioactivity with a diameter of $1.6 \mathrm{~mm}$. The nonuniform distribution of the activity along the circumference is also confirmed in the images. Outside the annulus, a tiny spot of activity is evident and appreciated at all exposure times, even as low as $0.1 \mathrm{~s}$. The size of the image of this spot was measured at $60 \mu \mathrm{m}$ (FWHM) on the image shown in Figure 2B. The actual size of the spot is unknown, but if it were a true point, this result would give an overall system resolution of $60 \mu \mathrm{m}$.
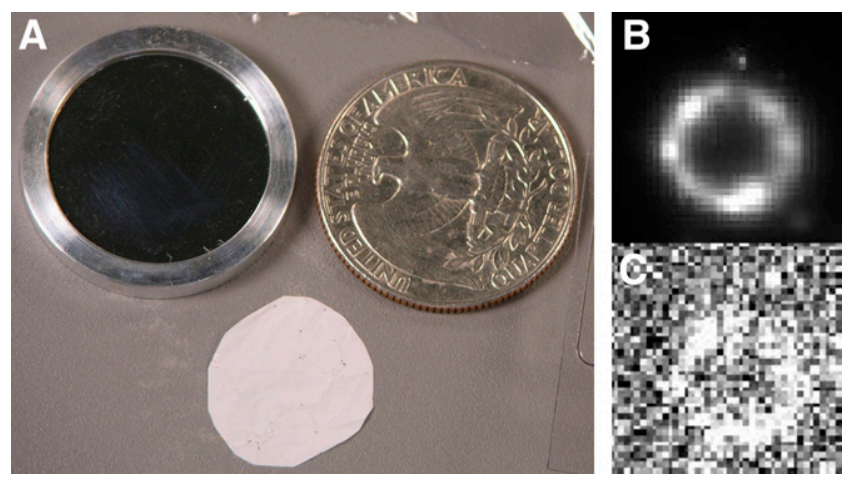

FIGURE 2. (A) Photograph showing point $\beta$ source on left, ultrathin phosphor at bottom, and quarter on right for size comparison. Images of $\beta$ source at 5 -min exposure (B) and 0.1 -s exposure $(\mathrm{C})$. 


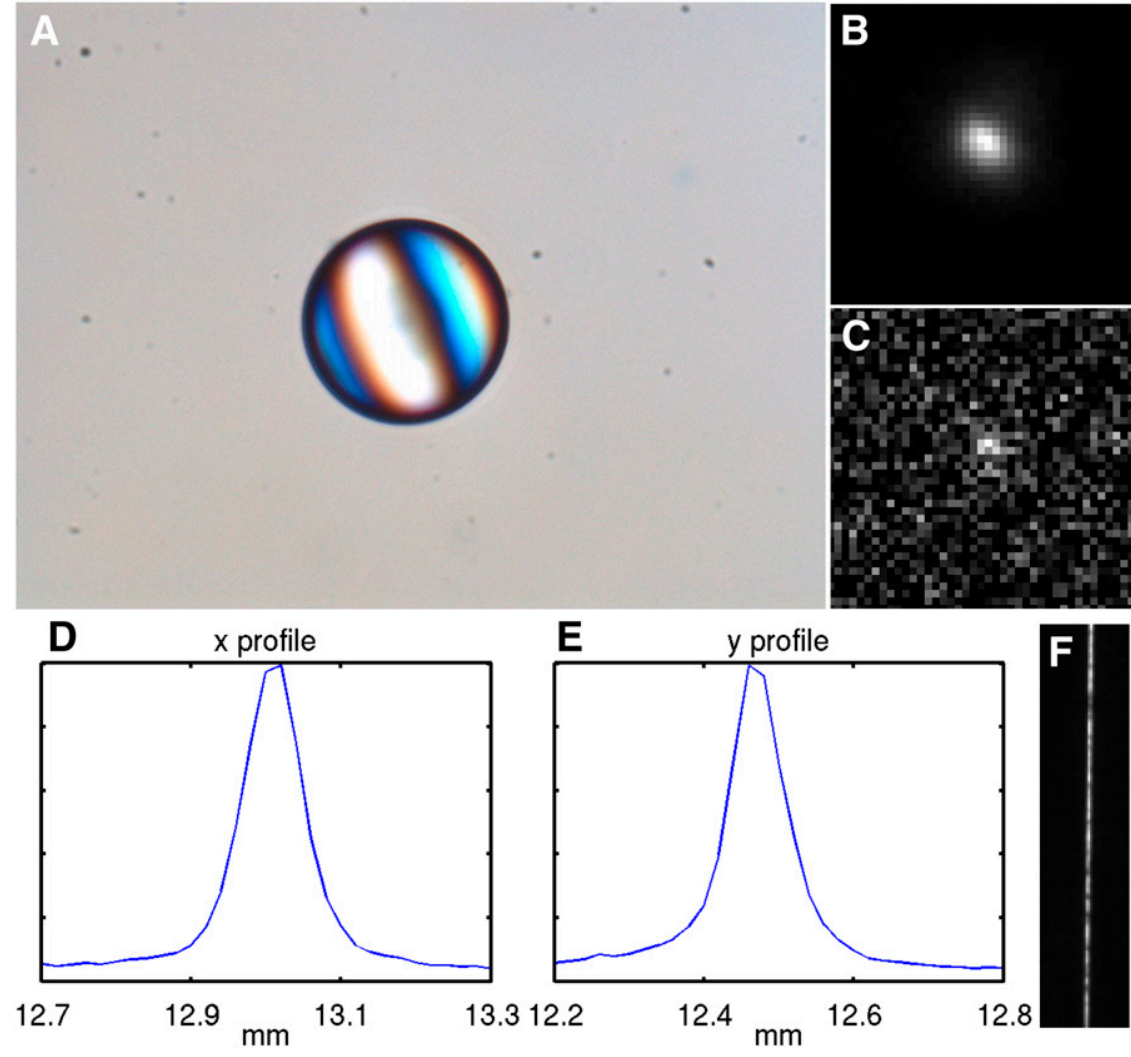

FIGURE 3. (A) Micrograph of ion-exchange resin bead labeled with $99 \mathrm{mTc}$ pertechnetate. Conversion-electron images of bead at 10-min exposure (B) and 2.5-s exposure (C). Profiles of conversion-electron image in $x$ direction $(\mathrm{D})$ and $y$ direction (E). (F) Optical image of $10-\mu \mathrm{m}$ slit using the same imaging components.
The conversion-electron image of the ${ }^{99 \mathrm{~m}} \mathrm{Tc}$-labeled resin bead is shown in Figure 3B. The FWHM of the profile of the electron image is about $90 \mu \mathrm{m}$ in both $x$ and $y$ directions (Figs. 3D and 3E). A micrograph of the same dried bead was taken with a regular microscope (Fig. 3A), on which the bead measures $70 \mu \mathrm{m}$ across. Because the bead diameter is much larger than the phosphor thickness, the conversion electrons from the bead have spread wider than $70 \mu \mathrm{m}$ and distributed more densely in the central area than on the outer rims when they reach the phosphor. The bead actually created an electron disk source with a complicated profile wider than $70 \mu \mathrm{m}$. If the FWHM of the disk were $70 \mu \mathrm{m}$, the result would also give a consistent overall system resolution of $50 \mu \mathrm{m}$, corresponding to $2.5 \mathrm{CCD}$ pixels.

An optical image of the narrow slit produced by the system is given in Figure 3F. The FWHM of the profile of the optical image is about $30 \mu \mathrm{m}$, which is a measurement of the optical resolution of the lens assembly and CCD camera.

\section{Sensitivity}

The system can produce a usable image of the ${ }^{90} \mathrm{Y} /{ }^{90} \mathrm{Sr}$ source at exposures as short as $0.1 \mathrm{~s}$, shown in Figure 2C. Only approximately $370 \beta$ particles are emitted from the source in $0.1 \mathrm{~s}$, of which 185 particles are emitted in the direction of the phosphor. Of these, fewer than 10 electrons are emitted from the tiny spot mentioned above, yet this spot is clearly identifiable.
An image of the ${ }^{99 \mathrm{~m}} \mathrm{Tc}$-labeled resin bead with a $2.5-\mathrm{s}$ exposure is shown in Figure 3C. Prior experiments in our laboratory (19) determined that most of the electron image exposure using a ${ }^{99 \mathrm{~m}} \mathrm{Tc}$ source arises from the $120-\mathrm{keV}$ conversion electrons produced in $10 \%$ of ${ }^{99 \mathrm{~m} T c}$ decays. The bead carried $814 \mathrm{~Bq}$ of activity; hence, during the 2.5 -s exposure, there were about 2,000 disintegrations and 2,000 high-energy conversion electrons, only half of which move toward the phosphor. Even at this exposure, the signal from the bead is still visible.

\section{In Vivo Imaging}

The conversion-electron images of the ${ }^{99 \mathrm{~m}} \mathrm{Tc}$ uptake in the mandibular glands of a normal mouse are presented in Figure 4. On the 5-min image, the gland is seen in sharp relief to the background technetium uptake in normal
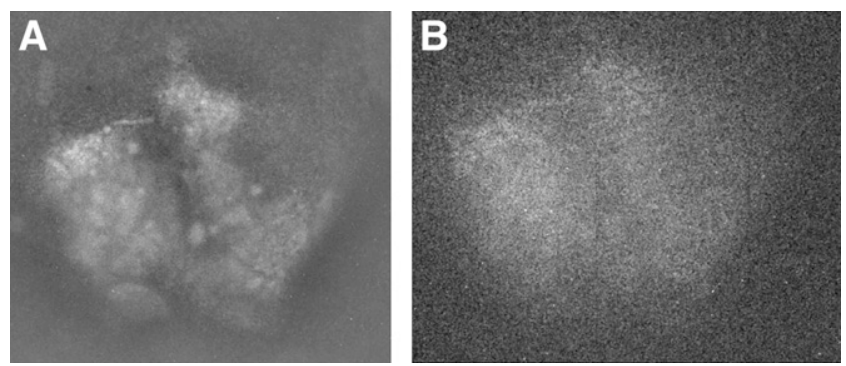

FIGURE 4. Images of mandibular glands of normal mouse for exposure times of $5 \mathrm{~min}(\mathrm{~A})$ and $30 \mathrm{~s}(\mathrm{~B})$. 
surrounding tissue. Many fine details within the gland are also clearly visible, indicating that, at least for the superficial few millimeters of gland reflected in the image, there is a markedly nodular uptake of technetium 30 min after injection. In another experiment on this animal days earlier, an internal jugular catheter had inadvertently lacerated the superior lateral margin of the left gland. This area is seen as an artifact from the earlier experiment. Even the 30-s image reflects the separation between the 2 glands and the laceration.

\section{DISCUSSION}

An energetic electron scatters very little when traversing a 3 - $\mu \mathrm{m}$-thick phosphor, and the thinness of the phosphor also reduces the scattering of the excited light. As currently configured, the optics and the pixel size on the CCD camera introduce about $30 \mu \mathrm{m}$ of blur, but numerous methods exist to improve this figure. Cameras with smaller pixels can be used, or the lenses can be used to magnify the image of the phosphor onto the camera. Even a microscope could be used to obtain the full intrinsic resolution of the 3- $\mu \mathrm{m}$ phosphor, albeit with a much reduced field of view. We estimate from Monte Carlo studies (to be published separately) that the intrinsic resolution of the phosphor with perfect optics would be about $10 \mu \mathrm{m}$.

When we image thick objects such as organs or tumors, some electrons from the activity deep in the object will reach and interact with the phosphor. These deep electrons will have scattered significantly within the object and deviated far from their original locations, reducing the overall spatial resolution compared with our measurements using relatively thin radioactive sources. The resolution in each experiment will then depend on the object thickness and composition.

The lens assembly has a large aperture, and the CCD is cooled to reduce its electronic noise. The entire system is therefore sensitive, even though only a small portion of the electron energy is deposited in an ultrathin phosphor. High sensitivity is critical for in vivo applications, because animals can be anesthetized for only a few hours without injury or perhaps alteration of normal physiology. The system sensitivity does not depend on the energy of an electron because an electron deposits only a small portion of its energy in the phosphor. When a phosphor is in contact with an object, the deposited energy at the beginning is approximately the same for electrons of different energies. The 3- $\mu \mathrm{m}$ Mylar substrate does not reduce the sensitivity because the signal with the phosphor side up is basically the same as that with the Mylar side up.

When imaging $\gamma$ emitters such as ${ }^{99 \mathrm{~m} T c}$, most of the signal is from conversion electrons because $\gamma$ rays deposit little energy in an ultrathin phosphor. Imaging conversion electrons ensures high resolution and sensitivity of the system to $\gamma$ emitters. Therefore, the electron-imaging system can image many kinds of radioisotopes, opening the possibility for using most radiopharmaceuticals.
The ultrathin phosphor is flexible, lightweight, thin, and biologically inert. These characteristics mean that it can be placed in contact with the tissues, organs, or tumors of a living organism without interfering with biological functions or physiology. The rest of the system is detached from the phosphor by a sufficient working distance. Potential clinical applications include imaging the positrons produced by ${ }^{18}$ F-FDG uptake in human melanomas with superior resolution, perhaps identifying the proper surgical margins for resection of primary skin malignancies.

\section{CONCLUSION}

We have developed a sensitive high-resolution electronimaging system using an ultrathin phosphor and a lenscoupled CCD camera. The system is able to image $\beta$ particles and positrons, as well as conversion electrons from $\gamma$-emitting isotopes with little contamination from $\gamma$ rays, making the system applicable to most isotopes. It will provide researchers with a new method of screening potential therapeutic and diagnostic radiopharmaceuticals. The system can also be a valuable tool for studying the biodistribution and microdosimetry of radiopharmaceuticals.

\section{ACKNOWLEDGMENTS}

We thank Dr. Gail Stevenson for animal preparation. We also thank Pier Ingram and Julia Sakamoto for their assistance with system setup. We are also grateful to Ms. Corrie Thies for her help in proofreading and editing the manuscript. This work was funded by grant P41 EB002035 from the NIH and by a departmental internal grant.

\section{REFERENCES}

1. Zanevsky YV, Chemenko SP, Ivanov AB, et al. Use of a proportional chamber for quantitative and qualitative analysis of thin-layer radio-chromatograms. Nucl Instrum Methods Phys Res. 1978;153:445.

2. Van Eijk CW, Hollander RW, Van der Slui SM. An MWPC for beta radiography. Nucl Instrum Methods Phys Res A. 1988;273:764-766.

3. Charpak G, Derr'e J, Giomataris Y, Rebourgeard Ph. Micromegas, a multipurpose gaseous detector. Nucl Instrum Methods Phys Res A. 2002;478:26-36.

4. Nagayoshi T, Kuboa H, Miuchia K, et al. Development of $\mu$-PIC and its imaging properties. Nucl Instrum Methods Phys Res A. 2004;525:20-27.

5. Orbom A, Dahlbom M, Olafsen T, et al. 3D biodistribution and dosimetry within tumours using a silicon-strip beta imager for serial digital autoradiography [abstract]. J Nucl Med. 2006;47(suppl):33P.

6. Ott RJ, MacDonald J, Wells K. The performance of a CCD digital autoradiography imaging system. Phys Med Biol. 2000;45:2011-2027.

7. Prydderch ML, Waltham NJ, Turchetta R, et al. A $512 \times 512$ CMOS monolithic active pixel sensor with integrated ADCs for space science. Nucl Instrum Methods Phys Res. 2003;512:358-367.

8. Mettivier G, Montesi MC, Russo P. Tritium digital autoradiography with a Medipix2 hybrid silicon pixel detector. Nucl Instrum Methods Phys Res. 2004;516:554-563.

9. Karellas A, Liu H, Reinhardt C, Harris LJ, Brill AB. Imaging of radionuclide emissions with a low-noise charge-coupled-device. IEEE Trans Nucl Sci. 1993; 40:979-982.

10. Puertolas D, Piedigrossi D, Leutz H, Gys T, D'Ambrosio C. An ISPA camera for beta radiography. IEEE Trans Nucl Sci. 1996;43:2477-2487.

11. Vu NT, Chung YH, Yu ZTF, et al. Direct detection of beta particles on a microfluidic chip using position sensitive APDs. Nuclear Science Symposium Conference Record, 2006. Vol. 6. Piscataway, NJ: IEEE; 2006:3536-3539. 
12. Laniece P, Charon Y, Dumas S, et al. HRRI: a high resolution radioimager for fast, direct quantification in in situ hybridization experiments. Biotechniques. 1994; 17:338-345.

13. Ljunggren K, Strand SE. Reduction of noise in the beta-camera for low activity applications. IEEE Trans Nucl Sci. 1994;41:1666-1667.

14. Kanno S, Rai H, Ohya T, Hayashi Y, Tanoi K, Nakanishi TM. Real-time imaging of radioisotope labeled compounds in a living plant. J Radioanal Nucl Chem. 2007;272:565-570

15. Hoffman EJ, Tornai MP, Levin CS, MacDonald LR, Siegel S. Gamma and beta intra-operative imaging probes. Nucl Instrum Methods Phys Res A. 1997;392: $324-329$.
16. Tornai MP, MacDonald LR, Levin CS, Siegel S, Hoffman EJ. Design considerations and initial performance of a $1.2 \mathrm{~cm}^{2}$ beta imaging intra-operative probe. IEEE Trans Nucl Sci. 1996;43:2326-2335.

17. Tornai MP, Patt BE, Iwanczyk JS, Tull CR, MacDonald LR, Hoffman EJ. A novel silicon array designed for intraoperative charged particle imaging. Med Phys. 2002;29:2529-2540.

18. Kroto SM. The Arizona Box: for Multiple Modality Luminescence Imaging [master's thesis]. Tucson, Arizona: University of Arizona; 2004.

19. Chen L, Gobar LS, Stevenson GD, Gmitro AF, Barrett HH. Electron imaging system using ultra-thin phosphor film and CCD camera for in vivo imaging [abstract]. J Nucl Med. 2007;48(suppl):47P-48P. 